\title{
Amplifiers in Tinnitus Patients
}

Henk M. Koning ${ }^{1 *}$

Bas C. ter Meulen²

\begin{abstract}
Introduction: High tinnitus loudness can be provoked by peripheral disorders of the somatosensory and/or auditory system.

Objectives: The object of our study was to compare high tinnitus loudness patients with low tinnitus loudness patients and to find specific factors associated with high tinnitus loudness.

Design: A retrospective cohort analysis of 234 patients with tinnitus as main complaint who visited our clinic in a four-year period.

Methods: Data obtained from the subjects were age, sex, the loudness of tinnitus estimated by the VAS, and the outcomes of the audiogram and the cervical spine radiograph.

Results: High tinnitus loudness was associated with a higher prevalence of females, more dizziness, less self-perceived hearing loss, more cervical disc degeneration at C3 to C6, and a larger anterior spur of cervical vertebrae C3 to C6. Females had a lower prevalence of tinnitus but a higher chance on high tinnitus loudness. Gender, the size of the largest anterior spur from C3 to C6, and the ratio of hearing loss at $8 \mathrm{kHz}$ and hearing loss at $2 \mathrm{kHz}$ are involved in the amplification of tinnitus loudness.

Conclusions: High tinnitus loudness can be provoked by peripheral disorders of the somatosensory and/or auditory system. A steep audiometric edge between hearing at $2 \mathrm{kHz}$ and hearing at $8 \mathrm{kHz}$ and/or cervical spine pathology with sympathetic nervous system irritation can amplify tinnitus loudness causing high tinnitus loudness.
\end{abstract}

Keywords: Tinnitus; Loudness; Cervical spine; Audiogram; Gender. 


\section{INTRODUCTION}

An intrigued question in clinical practice is why some patients have high tinnitus loudness while others have just a sound in their ear without any distress 1. This phenomenon can be explained by sensitisation of the auditory nervous system. Sensitisation is defined as an amplification of neural signalling within the nervous system. Sensitisation is categorized in peripheral and central sensitisation ${ }^{1-3}$. Peripheral sensitization is due to lowering of the activation threshold for physiological stimuli in the peripheral nerves. Central sensitization enhances the stimulus response of neural pathways in spinal cord and brain. Persistent peripheral sensitization leads to modifications further up in the nervous system which can subsequently end in central sensitization ${ }^{4}$. In spite of the fact that the site of the initial neural changes is distinguishable in peripheral and central sensitization, the final consequence of both is an increase in tinnitus loudness. Our hypothesis is that high tinnitus loudness can be provoked by peripheral disorders of the somatosensory and/or auditory system. The object of our study was to test this hypothesis by comparing high tinnitus loudness patients with low tinnitus loudness patients and to find specific factors associated with high tinnitus loudness.

\section{MATERIALS AND METHODS}

The Medical Ethics Committees United (Nieuwegein, the Netherlands) agreed this observational retrospective study (W20.240, October 14, 2020).

Subjects: All persons who came forward to our clinic for tinnitus in a four years period (1/10/2016 - 1/11/2020) took part in the study. The work-up of patients being a uniform clinical history, the loudness of tinnitus, an audiogram, and a cervical spine radiograph. The loudness of tinnitus was determined by the Visual Analogue Scale (VAS).
Data Assessment: Data obtained from the subjects were age, sex, the loudness of tinnitus estimated by the VAS, and the outcomes of the audiogram and the cervical spine radiograph. The VAS of tinnitus is a $10-\mathrm{cm}$ line between at the left end by "no tinnitus" and at the right end by "unbearable tinnitus". The patient indicates with marks the level of the mean, minimal and maximal perceived loudness of his/her tinnitus. The score is measured by the distance (millimetre) on the 10-cm line between the "no tinnitus" anchor and the patient's mark. High tinnitus loudness was defined as a maximal loudness of tinnitus above $73 \mathrm{~mm}$ and low tinnitus loudness as a maximal loudness of tinnitus of $73 \mathrm{~mm}$ or less $^{5}$. An audiogram evaluated six pure tone boundaries from $250 \mathrm{~Hz}$ to $8 \mathrm{kHz}$. The most powerful side of the tinnitus was chosen for research. In instances of tinnitus with similar loudness at each side, the average of both outcomes was chosen for research. Measurements of the cervical spine radiographs included the corner between the posterior side of the third and sixth cervical vertebrae, the intervertebral disc height from the second till the seventh cervical vertebrae, and the dimension of the anterior spur of the third till the sixth cervical vertebrae, as previously described ${ }^{6}$.

Statistical Methods: Statistical analysis was executed with Minitab 18 (Minitab Inc., State College, PA, USA). Student's t-test was employed for continuous variables and $\mathrm{X} 2$ test for dichotomous variables. Discriminant analysis was used in order to evaluate the occurrence of high loudness tinnitus with the combination of gender, the magnitude of the largest anterior spur from C3 to C6, and the hearing deficit at $8 \mathrm{kHz}$ compared to the hearing deficit at $2 \mathrm{kHz}$. A value of $P$ less than 0.05 was statistically significant.

\section{RESULTS}

In a four-year period, 234 patients approached our clinic for advice concerning their tinnitus. The hallmarks of these subjects are shown in Table 1. Tinnitus was linked with

Table 1: Clinical characteristics of the patients with tinnitus.

\begin{tabular}{|c|c|c|c|}
\hline & Prevalence & Median & Q1-Q3 \\
\hline Gender (Male) & $57 \%$ & & \\
\hline Self perceived hearing loss & $62 \%$ & & \\
\hline Cervical pain & $64 \%$ & & \\
\hline Age (year) & & 57 & $50-63$ \\
\hline \multicolumn{4}{|l|}{ Hearing loss $(\mathrm{dB})$ at: } \\
\hline$-250 \mathrm{~Hz}$ & & 15 & $8-25$ \\
\hline$-500 \mathrm{~Hz}$ & & 15 & $5-25$ \\
\hline$-1 \mathrm{KHz}$ & & 15 & $10-30$ \\
\hline$-2 \mathrm{KHz}$ & & 20 & $10-40$ \\
\hline$-4 \mathrm{KHz}$ & & 40 & $20-55$ \\
\hline$-8 \mathrm{KHz}$ & & 50 & 2866 \\
\hline \multicolumn{4}{|c|}{ Intensity of the tinnitus (VAS; millimetre) } \\
\hline - Mean: & & 68 & 5082 \\
\hline - Minimal: & & 40 & 1860 \\
\hline - Maximal: & & 86 & 7294 \\
\hline
\end{tabular}

dB: decibel; Hz: Hertz; KHz: Kilohertz; VAS: visual analogue scale; Q1 - Q3: inter-quartile range. 
a widespread presence of self- perceived hearing loss, cervical pain, and hearing deficit in the higher frequency $(4 \mathrm{kHz}$ to $8 \mathrm{kHz})$. Patients with low tinnitus loudness were compared with patients with high loudness tinnitus (Table 2). High tinnitus loudness was associated with a higher prevalence of females and dizziness, more self-perceived hearing loss, more cervical disc degeneration at $\mathrm{C} 3$ to $\mathrm{C} 6$, and larger anterior spurs, especially at $\mathrm{C} 4$. Females had a lower prevalence of tinnitus but a higher chance on high loudness tinnitus. The size of the largest anterior spur between C3 and $\mathrm{C} 6$ above $19 \%$ increases the prevalence of high tinnitus loudness from 64 to $82 \%(p=0.013)$. Multivariate statistical analysis indicates that gender, the size of the largest anterior spur from $\mathrm{C} 3$ to $\mathrm{C} 6$, and the ratio of hearing loss at $8 \mathrm{kHz}$ and hearing loss at $2 \mathrm{kHz}$ could predict the occurrence of high tinnitus loudness the best (Proportion Correct $=0.664$. With discriminant analysis, the patient group with a higher chance on high tinnitus loudness was identified (Figure 1).

Table 2: Patients with high tinnitus loudness were compared with low tinnitus loudness patients.

\begin{tabular}{|c|c|c|c|c|c|c|c|c|}
\hline & \multicolumn{3}{|c|}{$\begin{array}{l}\text { High tinnitus loudness } \\
\qquad(\mathrm{n}=111)\end{array}$} & \multicolumn{3}{|c|}{$\begin{array}{l}\text { Low tinnitus loudness } \\
\qquad(\mathrm{n}=41)\end{array}$} & \multirow[t]{2}{*}{ P-value } & \multirow[b]{3}{*}{$\mathrm{S}$} \\
\hline & Prev. & Mean & SEM & Prev. & Mean & SEM & & \\
\hline Gender (Male) & $51 \%$ & & & $76 \%$ & & & 0.007 & \\
\hline Postural instability & $42 \%$ & & & $29 \%$ & & & 0.142 & \\
\hline Dizziness & $41 \%$ & & & $22 \%$ & & & 0.034 & $\mathrm{~S}$ \\
\hline Self-perceived hearing loss & $58 \%$ & & & $78 \%$ & & & 0.024 & S \\
\hline Cervical pain & $62 \%$ & & & $56 \%$ & & & 0.497 & \\
\hline Age at the start of the complaint (year) & & 47 & 1.3 & & 46 & 2.0 & 0.693 & \\
\hline \multicolumn{9}{|l|}{ Hearing loss $(\mathrm{dB})$ at: } \\
\hline$-\quad 250 \mathrm{~Hz}$ & & 19 & 1.9 & & 16 & 2.4 & 0.410 & \\
\hline $500 \mathrm{~Hz}$ & & 20 & 2.0 & & 17 & 2.5 & 0.413 & \\
\hline $1 \mathrm{kHz}$ & & 21 & 2.0 & & 20 & 2.8 & 0.788 & \\
\hline $2 \mathrm{kHz}$ & & 24 & 2.0 & & 31 & 7.6 & 0.386 & \\
\hline $4 \mathrm{kHz}$ & & 42 & 2.3 & & 37 & 3.4 & 0.303 & \\
\hline $8 \mathrm{kHz}$ & & 51 & 2.6 & & 45 & 4.0 & 0.178 & \\
\hline Angle between vertebrae C2 and C6 (degrees) & & 5 & 0.9 & & 9 & 1.6 & 0.056 & \\
\hline \multicolumn{9}{|l|}{ Farfan's measurement of disc space height (\%): } \\
\hline$-\quad$ C2-C3: & & 38 & 0.7 & & 40 & 1.1 & 0.277 & \\
\hline C3-C4: & & 35 & 0.8 & & 38 & 1.1 & 0.037 & $\mathrm{~S}$ \\
\hline C4-C5: & & 33 & 0.8 & & 36 & 0.9 & 0.013 & S \\
\hline C5-C6: & & 25 & 0.8 & & 29 & 1.2 & 0.013 & $\mathrm{~S}$ \\
\hline$-\quad$ C6-C7: & & 27 & 0.9 & & 27 & 1.6 & 0.926 & \\
\hline \multicolumn{9}{|l|}{ Size of anterior osteophyte (\%): } \\
\hline$-\quad \mathrm{C} 3$ & & 8 & 0.5 & & 7 & 0.6 & 0.176 & \\
\hline $\mathrm{C} 4$ & & 12 & 0.7 & & 9 & 0.9 & 0.018 & $\mathrm{~S}$ \\
\hline C5 & & 19 & 0.8 & & 17 & 1.0 & 0.061 & \\
\hline$-\quad \quad$ C6 & & 14 & 0.6 & & 13 & 1.1 & 0.259 & \\
\hline Largest size anterior osteophyte from C3 to C6 & & 21 & 0.8 & & 18 & 1.0 & 0.016 & $\mathrm{~S}$ \\
\hline
\end{tabular}

dB: decibel; Hz: Hertz; KHz: Kilohertz; Prev.: Prevalence; SEM: Standard Error of the Mean; mm: millimetre; S: Significant

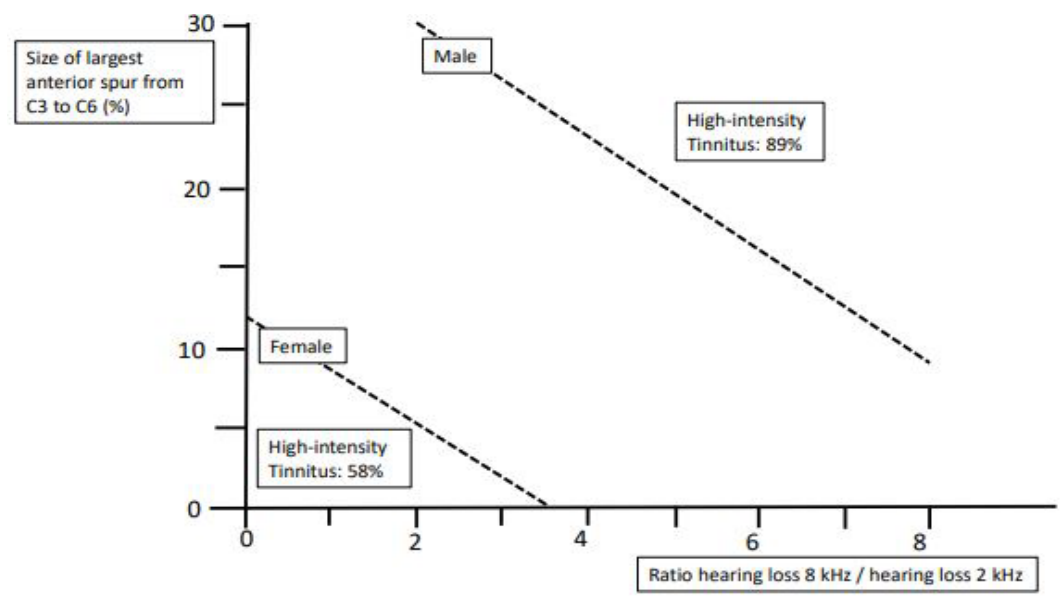

Figure 1: The chance for high tinnitus loudness depends on gender, the magnitude of the largest anterior spur from C3 to C6, and the ratio of hearing loss at $8 \mathrm{kHz}$ and hearing loss at $2 \mathrm{kHz}$. For example, in a male with an anterior spur of the cervical spine from C3 to C6 of 15\% and a ratio of hearing loss $8 \mathrm{kHz} /$ hearing loss $2 \mathrm{kHz}$ of 4, the chance on high tinnitus loudness is $58 \%$, but for a female the chance is $89 \%$. 
Patients fulfilling these criteria, $89 \%$ of them had high tinnitus loudness compared to $58 \%$ in patients not fulfilling these criteria.

\section{DISCUSSION}

High tinnitus loudness was associated with a higher prevalence of females, more dizziness, less self-perceived hearing loss, more cervical disc degeneration at $\mathrm{C} 3$ to C6, and a larger anterior spur of cervical vertebrae C3 to C6. Females had a lower prevalence of tinnitus but a higher chance on high tinnitus loudness. Gender, the size of the largest anterior spur from C3 to $\mathrm{C} 6$, and the ratio of hearing loss at $8 \mathrm{kHz}$ and hearing loss at $2 \mathrm{kHz}$ are involved in the amplification of tinnitus loudness. Tinnitus is an acoustic percept which can be subdivide into tinnitus loudness and tinnitus distress ${ }^{7}$. Tinnitus distress and tinnitus loudness are independent clinical factors $^{8}$. The brain possesses a network that reproduce the perceived tinnitus loudness. This network is related to the default mode network, consisting of the anterior cingulate cortex, the insula, the parahippocampus, and the auditory cortex 8 . Tinnitus loudness can be estimated by ratings on a VAS $^{9}$. A perceived maximal loudness of the tinnitus above 73 millimetres is linked to an increased prevalence of difficult psychosocial situations. Therefore, we use this as limit for high tinnitus loudness. Sensitization is an increased response of the nervous system to normal or subthreshold afferent input ${ }^{10}$. It can be divided into peripheral and central sensitisation. Peripheral sensitization indicates that the peripheral afferent neurons become sensitive due to local tissue injury or inflammatory processes, thus amplifying neural signals ${ }^{11}$. Central sensitization is defined as an amplification of neural signalling within the central nervous system. There can be a two-way interaction between peripheral sensitization and central sensitization: central sensitization is not only consequential to peripheral sensitization but may, in turn, adjust peripheral sensitization ${ }^{12}$. Therefore, understanding tinnitus loudness involves the identification of peripheral and central contributory factors for sensitisation in the auditory pathway. Tinnitus conceptually involves not only neuroplasticity in the central nervous system tinnitus but also sensitization in primary sensory neurons of dorsal root ganglia, trigeminal ganglia, and/or the cochlea ${ }^{13,14}$. An altered afferent signal of the peripheral auditory or somatosensory system results in the gener $\neg$ ation of tinnitus in the central auditory system. Persistent perception of tinnitus may no longer be coupled to the intensity or even the presence of the noxious peripheral stimuli ${ }^{4}$. We found that gender, the size of the largest anterior spur from $\mathrm{C} 3$ to $\mathrm{C} 6$, and the ratio of hearing loss at $8 \mathrm{kHz}$ and hearing loss at $2 \mathrm{kHz}$ are involved in the amplification of tinnitus loudness. In our study, a higher hearing loss at $8 \mathrm{kHz}$ compared to the hearing loss at 2 $\mathrm{kHz}$ is associated with higher tinnitus loudness. A sloping high frequency hearing deficit is found in sensory and mechanical presbycusis which can be due to damage to outer hair cells or from stiffening of the basilar membrane of the cochlea ${ }^{15}$. A steep audiometric edge between regions of normal and impaired hearing may be sufficient to disrupt the normal pattern of neural synchrony in tonotopically organized regions of the central auditory system $^{16,17}$. A cochlear amplifier impairment due to a loss of outer hair cells is also coupled to structural cerebral brain modifications ${ }^{9}$. The cingulate area and the parahippocampus gyrus are crucial elements of the default mode network which were importantly wasted in patients with cochlear amplifier disorder. These areas in the default mode network are also implicated in the sensation of tinnitus loudness ${ }^{8}$. A high frequency hearing loss might induce changes in the default mode network resulting in high tinnitus loudness. The superior cervical ganglion and the stellate ganglion are the origin of the sympathetic nerves who innervates the cochlea and regulates cochlear blood flow via the local adrenergic $\alpha$-receptors ${ }^{18}$. Pathological alterations of the cervical intervertebral disc, such as large anterior spurs, may irritate sympathetic nerve fibers causing reduced cochlear blood flow and an increase of tinnitus loudness ${ }^{19}$. Large anterior cervical spurs are also linked to a better result of treatment of the superior cervical sympathetic ganglion to reduce tinnitus loudness 6 . Cervical spine degeneration with stimulating of the sympathetic nervous system can result in an amplification of tinnitus loudness. Females had a lower prevalence of tinnitus but a higher chance on high loudness tinnitus. It is already known that men have more likelihood of developing tinnitus and presbycusis than women ${ }^{20,21}$. The sex inequality is believed to be in some extent related to shifts in ovarian steroid hormones, although the exact mechanisms are not yet completely understood 22. Estrogens might possess potent antioxidant properties and exert neuroprotective actions $^{19-22}$. However, we could find no explanation for the higher chance on high loudness for women. We note some limitations in this study. Tinnitus loudness assessments rely to a great extent on the method of measurement used. Tinnitus loudness can be valued in a technique in which the intensity of an exterior sound is set side by side to the observed tinnitus loudness. Tinnitus loudness can also be established by rankings on a VAS. Tinnitus loudness assessed by VAS rating correlate better with tinnitus distress and is easier to access ${ }^{23}$. Also, this study is limited by a selection bias. There might be an overestimation of patients with high tinnitus loudness. Patients coming to our clinic want a reduction of their tinnitus loudness and thus there is a greater chance that they have high tinnitus loudness. Another limitation is its retrospective kind and a prospective follow-up study is advocated to validate these results and the conclusions.

\section{CONCLUSION}

Gender, the magnitude of the largest anterior spur from $\mathrm{C} 3$ to $\mathrm{C} 6$, and the ratio of hearing loss at $8 \mathrm{kHz}$ and hearing loss at $2 \mathrm{kHz}$ are involved in the amplification of tinnitus loudness. Females had a lower prevalence of tinnitus but a higher chance on high tinnitus loudness. High tinnitus loudness can be provoked by peripheral disorders of the somatosensory and/or auditory system. 
A steep audiometric edge between hearing at $2 \mathrm{kHz}$ and hearing at $8 \mathrm{kHz}$ and/or cervical spine pathology with sympathetic nervous system irritation can amplify tinnitus loudness causing high tinnitus loudness.

\section{CONFLICT OF INTEREST}

The authors declares no conflict of interest.

\section{REFERENCES}

1. Balkenhol T, Wallhäusser-Franke E, Delb W. Psychoacoustic tinnitus loudness and tinnitus-related distress show different associations with oscillatory brain activity. PLoS One. 2013;8:e53180.

2. Woolf CJ. Central sensitization: implications for the diagnosis and treatment of pain. Pain. 2011;152:2-15.

3. Monaco A, Cattaneo R, Marci MC, Pietropaoli D, Ortu E. Central Sensitization-Based Classification for Temporomandibular Disorders: A Pathogenetic Hypothesis. Pain Res Manag. 2017;59:570-6.

4. Fleming KC, Volcheck MM. Central sensitization syndrome and the initial evaluation of a patient with fibromyalgia: a review. Rambam Maimonides Med J. 2015;6:10-20.

5. Koning HM. Psychosocial consequences of the loudness of tinnitus. Int Tinnitus J. 2019;23:103-7.

6. Koning HM, Koning MV, Koning NJ, ter Meulen BC. Anterior Cervical Osteophytes and Sympathetic Hyperactivity in $\mathrm{Pa}-$ tients with Tinnitus: Size Matters. Int Tinnitus J. 2018;22:97102.

7. Ghodratitoostani I, Zana Y, Delbem AC, Sani SS, Ekhtiari H, Sanchez TG. Theoretical Tinnitus Framework: A Neurofunctional Model. Front Neurosci. 2016;10:370-2.

8. Ueyama, T, Donishi T, Ukai S, Ikeda Y, Hotomi M, Yamanaka $\mathrm{N}$, et al. Brain regions responsible for tinnitus distress and loudness: a resting-state FMRI study. PLoS One. 2013;8:677-8.

9. Belkhiria, C, Vergara RC, San Martín S, Leiva A, Marcenaro B, Martinez M, et al. Cingulate Cortex Atrophy Is Associated With Hearing Loss in Presbycusis With Cochlear Amplifier Dysfunction. Front Aging Neurosci. 2019;11:97-100.

10. Ji RR, Nackley A, Huh Y, Terrando N, Maixner W. Neuroinflammation and Central Sensitization in Chronic and Widespread Pain. Anesthesiology. 2018;129:343-66.
11. lyengar S, Ossipov MH, Johnson KW. The role of calcitonin gene-related peptide in peripheral and central pain mechanisms including migraine. Pain. 2017;158:543-59.

12. Mulders WH, Barry KM, Robertson D. Effects of furosemide on cochlear neural activity, central hyperactivity and behavioural tinnitus after cochlear trauma in guinea pig. PLoS One. 2014;9:979-88.

13. Sanchez TG, Rocha CB. Diagnosis and management of somatosensory tinnitus: review article. Clinics (Sao Paulo). 2011;66:1089-94.

14. Calderon PS, Hilgenberg PB, Rossetti LM, Laurenti JV, Conti PC. Influence of tinnitus on pain severity and quality of life in patients with temporomandibular disorders. J Appl Oral Sci. 2012;20:170-3.

15. Arvin B, Prepageran N, Raman R. "High frequency presbycusis" is there an earlier onset?. Indian J Otolaryngol Head Neck Surg. 2013;65:480-4.

16. Diesch E, Andermann M, Flor H, Rupp A. Functional and structural aspects of tinnitus-related enhancement and suppression of auditory cortex activity. Neurolmage. 2010;50:1545-59.

17. Haider HF, Bojic T, Ribeiro SF, Paço J, Hall DA, Szczepek AJ. Pathophysiology of Subjective Tinnitus: Triggers and Maintenance. Front Neurosci. 2018;12:866-8.

18. Bartolome MV, Gil-Loyzaga P. Serotonergic innervation of the inner ear: Is it involved in the general physiological control of the auditory receptor? Int Tinnitus J. 2005;11:119-25.

19. Wang Z, Wang X, Yuan W, Jiang D. Degenerative pathological irritations to cervical PLL may play a role in presenting sympathetic symptoms. Med Hypotheses. 2011;77:921-3.

20. Wang J, Puel JL. Presbycusis: An Update on Cochlear Mechanisms and Therapies. J Clin Med. 2020;9:218-20.

21. Profant $O$, Škoch $A$, Tintěra J, Svobodová $V$, Kuchárová D, Svobodová Burianová J, et al. The Influence of Aging, Hearing, and Tinnitus on the Morphology of Cortical Gray Matter, Amygdala, and Hippocampus. Front Aging Neurosci. 2020;12:553-61.

22. Al-Hassany L, Haas J, Piccininni M, Kurth T, Maassen VD, Rohmann JL. Giving Researchers a Headache - Sex and Gender Differences in Migraine. Front Neurol. 2020;11:549-68.

23. Kidd lii AR, Bao J. Recent advances in the study of age-related hearing loss: a mini-review. Gerontology. 2012;58:490-6. 